\title{
Universality classes for unstable crystal growth
}

\author{
Sofia Biagi, ${ }^{1,2, *}$ Chaouqi Misbah, ${ }^{1,2, \dagger}$ and Paolo Politi ${ }^{2,3, \ddagger}$ \\ ${ }^{1}$ Université Grenoble 1/CNRS, LIPhy UMR 5588, Grenoble, F-38401, France \\ ${ }^{2}$ Istituto dei Sistemi Complessi, Consiglio Nazionale delle Ricerche, \\ Via Madonna del Piano 10, 50019 Sesto Fiorentino, Italy \\ ${ }^{3}$ INFN Sezione di Firenze, via G. Sansone 1, 50019 Sesto Fiorentino, Italy
}

(Dated: January 20, 2021)

\begin{abstract}
Universality has been a key concept for the classification of equilibrium critical phenomena, allowing associations among different physical processes and models. When dealing with nonequilibrium problems, however, the distinction in universality classes is not as clear and few are the examples, as phase separation and kinetic roughening, for which universality has allowed to classify results in a general spirit. Here we focus on an out-of-equilibrium case, unstable crystal growth, lying in between phase ordering and pattern formation. We consider a well established $2+1$ dimensional family of continuum nonlinear equations for the local height $h(\mathbf{x}, t)$ of a crystal surface having the general form $\partial_{t} h(\mathbf{x}, t)=-\nabla \cdot\left[\mathbf{j}(\nabla h)+\nabla\left(\nabla^{2} h\right)\right]: \mathbf{j}(\nabla h)$ is an arbitrary function, which is linear for small $\nabla h$, and whose structure expresses instabilities which lead to the formation of pyramid-like structures of planar size $L$ and height $H$. Our task is the choice and calculation of the quantities that can operate as critical exponents, together with the discussion of what is relevant or not to the definition of our universality class. These aims are achieved by means of a perturbative, multiscale analysis of our model, leading to phase diffusion equations whose diffusion coefficients encapsulate all relevant informations on dynamics. We identify two critical exponents: i) the coarsening exponent, $n$, controlling the increase in time of the typical size of the pattern, $L \sim t^{n}$; ii) the exponent $\beta$, controlling the increase in time of the typical slope of the pattern, $M \sim t^{\beta}$ where $M \approx H / L$. Our study reveals that there are only two different universality classes, according to the presence $(n=1 / 3, \beta=0)$ or the absence $(n=1 / 4, \beta>0)$ of faceting. The symmetry of the pattern, as well as the symmetry of the surface mass current $\mathbf{j}(\nabla h)$ and its precise functional form, is irrelevant. Our analysis seems to support the idea that also space dimensionality is irrelevant.
\end{abstract}

PACS numbers: 05.70.Ln,81.10.Aj,05.45.-a

\section{INTRODUCTION: UNIVERSALITY CLASSES}

The concept of universality is very useful in physics, because it allows to classify seemingly different phenomena and models. Perhaps, one of the oldest examples is the universal form of the Van der Waals equation of state (law of corresponding states [1]), which is the simplest equation describing a change of state and valid for any fluid. A clear formalization of universality was firstly possible for equilibrium critical phenomena, where the renormalization group theory allows to give a rigorous definition of which parameters are relevant (universal) and which are not. For example, within important classes of ferromagnetic spin models, it is known that relevant parameters are: the physical dimension of the space, the dimension of the order parameter and its symmetries, the (short/long) range of interaction of the coupling. A universality class is uniquely defined by its critical exponents, which describe the behaviour of the order parameter in proximity of the critical point as a function either of the control parameter (e.g. the temperature) or of the conjugate field of the order parameter (e.g. the magnetic

\footnotetext{
* sofia.biagi@ujf-grenoble.fr

$\dagger$ chaouqi.misbah@ujf-grenoble.fr

$\ddagger$ paolo.politi@isc.cnr.it
}

field).

When passing to nonequilibrium processes, the phenomenology is much wider and a classification in universality classes is not as firm. A much studied case is the so-called "phase separation". Let us consider a system undergoing a continuous phase transition (at $T=T_{c}$ ) when passing from a disordered high temperature phase to an ordered low temperature phase. If the temperature $T$ is suddenly decreased (quenching) from $T_{i}>T_{c}$ to $T_{f}<T_{c}$, the system undergoes an ordering process where the typical size $L$ of ordered regions increases in time, $L(t)$. This process, called coarsening, lasts forever (for infinite systems) if the system is globally at thermodynamic equilibrium. In most cases $L(t)$ increases as a power law, $L(t) \sim t^{n}$, which defines the coarsening exponent $n$. Generally speaking, $T_{i}$ and $T_{f}$ are irrelevant parameters and it appears that the physical space dimension is also irrelevant (as long as $T_{c}$ is finite, $T_{c}>0$ ). It appears instead that conservation laws are relevant for the dynamics and it is reasonable to expect that a conservation law slows down the dynamics and reduces the coarsening exponent, a known fact at present [2].

The spirit of universality also means that the same model is important for different physical problems: for example, phase separation and pattern formation have several similarities. Therefore our study, which focuses on a certain class of growth equations for crystals exhibit- 
ing pattern formation, is expected to be relevant for both fields. This class of equations, see Eq. (3), has emerged in the last twenty years as a prototypical description of crystal growth by deposition processes. It has some similarities with well known models as the Cahn-Hilliard equation and the clock models [3], but its general properties have been now fully established, as discussed in the next section.

This equation leads to morphological instability of a planar surface, with formation of mounds/pyramids out of the flat front. In general, as previously sketched for domains in phase separation processes, mounds coarsen, but under some conditions we show that other scenarios take place. In apparent contrast to some existing literature (see Section VII A), we are able to state that pattern symmetry is irrelevant and only two universality classes result, depending on whether mound's slope is constant (faceting) or it is an increasing function of time. This last feature is known a priori, from visual inspection of the surface current $\mathbf{j}(\mathbf{m})$ (see below) and allows the definition of a second exponent $\beta \geq 0$, describing the behavior of the typical mound's slope $M$ in time, $M(t) \sim t^{\beta}$. The two universality classes we have found are therefore given, in the case of constant slope $\mathbf{m}^{*}\left(\mathbf{j}\left(\mathbf{m}^{*}\right)=0\right)$, by $\beta=0$ and $n=1 / 3$, and, in the case of increasing slope, by $\beta>0$ and $n=1 / 4$.

The idea used to establish these results is based on the statement that coarsening takes place if the steadystate periodic solutions are unstable against perturbations of the phase of the pattern [4]. More precisely, a periodic pattern has a constant wavenumber $\mathbf{q}$ which acquires a space-time dependence when the pattern is perturbed (we can also define the phase of the pattern): if the periodic pattern is perturbed, the wavenumber (as well as the phase) will vary from one point to another. If the perturbation grows with time, we say that the pattern is unstable with respect to wavenumber (or phase) fluctuations. If the periodic pattern is unstable with respect to phase fluctuations then we expect coarsening to take place. It will be shown that the phase of the pattern obeys a diffusion equation and instability is signaled by a negative diffusion coefficient $D$. This diffusion coefficient (actually in two dimensions there are several diffusion coefficients, as we shall see) depends on the steady-state pattern properties, and more particularly on the modulus of the wavenumber $q$. By using a dimensional relation, $|D(q)| \approx L^{2} / t$, where $q=2 \pi / L$, we shall extract the coarsening exponent.

Here we are able to make stronger and more general statements with respect to [5], facing a wider range of two-dimensional patterns and stressing on universal features of unstable crystal growth. This is the focus of the present paper: going beyond the details of the equation and of the physical process and pointing out what is relevant, slope selection or not, and what is not, the symmetry of the pattern and that of the mass current. Although no complete proof about physical space dimensionality is accomplished, our study reveals strong support regarding its irrelevance.

\section{CRYSTAL GROWTH EQUATION}

In this Section we shall give a brief introduction to the class of equations we are interested in, mainly addressing the qualitative aspects of the dynamics rather than their physical derivation (for a thorough discussion on the physical background, the reader is referred to [6]).

A growing planar crystal surface (growing by molecular beam epitaxy, for example) can undergo a morphological instability resulting into the formation of threedimensional mounds or pyramids of linear size $L$ and height $H$. The subsequent morphological evolution may range from a pattern of constant $L$ and an increasing $H$ up to a perpetual increase of $L$ in the course of time (coarsening), with $H$ increasing in concert. An intermediate scenario may also take place in some cases, where $L(t)$ increases up to a length $L_{\max }$ reached at a given time, beyond which the mound size is frozen, while mound height keeps growing. This scenario corresponds to interrupted coarsening [7]. We are not aware of a scenario where both $L$ and $H$ keep constant in time.

In the case of a perpetual coarsening the generic evolution law of $L(t)$ is algebraic with coarsening exponent, $n$, defined as $L(t) \sim t^{n}$. During the coarsening process, the typical slope $M \approx H / L$ may either keep constant or increase in time, $M(t) \sim t^{\beta}$, therefore defining a second exponent $\beta \geq 0$.

From a mesoscopic point of view, the local velocity $\partial_{t} z$ of a surface $z(\mathbf{x}, t)$ growing under a deposition flux of intensity $F_{0}$ must have the form

$$
\partial_{t} z(\mathbf{x}, t)=F_{0}-\nabla \cdot \mathbf{J}_{t o t},
$$

provided that the deposited mass on the surface does not evaporate and that no holes occur in the growing solids [8]. The total current $\mathbf{J}_{t o t}$ is a function of the slope $\mathbf{m}=\nabla z$ and higher order spatial derivatives and it accounts for all surface rearrangement processes. Its simplest form is

$$
\mathbf{J}_{t o t}=\mathbf{j}(\mathbf{m})+\Gamma \nabla^{2} \mathbf{m},
$$

where $\mathbf{j}(\mathbf{m})$ is a function of the slope only and it accounts for the existence of a mass current on a terrace. At small slopes $\mathbf{j} \approx \nu \nabla z$ : if the current is uphill $(\nu>0)$, the flat surface is destabilized at sufficiently large scales. The second term, $\nabla^{2} \mathbf{m}$, regularizes the dynamics at short length scales and it may have different physical origins [9].

By performing the substitution $z \rightarrow h=z-F_{0} t$ and after appropriate rescaling of $\mathbf{x}$ and $t$, it is possible to absorb $\Gamma$ and $\nu$ into the new variables so that the equation can be written in the form

$$
\frac{\partial h(\mathbf{x}, t)}{\partial t}=-\nabla \cdot\left[\mathbf{j}(\nabla h)+\nabla\left(\nabla^{2} h\right)\right] \equiv-\nabla \cdot \mathbf{J}_{t o t},
$$


where $\mathbf{j}(\nabla h)=\nabla h+$ higher order terms.

Some important features of the nonlinear dynamics can be discussed by referring to the one dimensional version of Eq. (3), which has been discussed at length in Ref. [4]:

$$
\partial_{t} h=-\partial_{x}\left[j\left(h_{x}\right)+h_{x x x}\right]
$$

In fact, by taking the spatial derivative of both sides, we get the generalized Cahn-Hilliard equation,

$$
\partial_{t} m=-\partial_{x x}\left[j(m)+m_{x x}\right]
$$

where the shape of the potential $U(m)=\int d m j(m)$ determines the type of dynamics [10]: (i) stationary solutions, satisfying $j(m)+m_{x x}=0$, correspond to periodic "oscillations" within the potential well $U(m)$; (ii) there is coarsening if and only if the wavelength of such stationary solutions is an increasing function of their amplitude; in general three scenarios, depicted here above, are possible: perpetual coarsening, interrupted coarsening, no coarsening; (iii) the slope of emerging mounds is constant if $U(m)$ has maxima at finite $m= \pm m^{*}$, otherwise slope increases forever.

When passing from one to two dimensions, i.e. passing from Eq. (4) to Eq. (3), the equivalence between the growth equation and the Cahn-Hilliard equation ceases to be valid [6] (see also Section VII). Furthermore, the surface current $\mathbf{j}$ requires specification of its in-plane symmetry, which adds a new degree of freedom to the problem. The following Sections present the various dynamical scenarios where the values of the exponents $n$ and $\beta$ (universality classes) are extracted for the family of models defined by Eq. (3). We shall follow a multiscale perturbative approach, discussed in the next Section, which allows us to write down the phase diffusion equation that describes the evolution of the typical mound size in the course of time. The various dynamical scenarios will constitute the subject of Sec. IV while distinct universality classes will presented in Sec. VI. A thorough discussion of our results will follow in Sec. VII.

\section{THE PHASE DIFFUSION EQUATION}

As already anticipated in the previous Section, the flat profile, namely the solution $h \equiv 0$ of Eq. (3), is unstable. This is easily shown from a linear stability analysis: setting $h=\delta \exp (\omega t+i \mathbf{k} \cdot \mathbf{x})$ in Eq. (3) and assuming $\delta \ll 1$, we obtain the linear spectrum:

$$
\omega(k)=k^{2}-k^{4}
$$

where $k=|\mathbf{k}|$. This result shows that there is a band of wave-vectors $(0<k<1)$ with positive $\omega$, so the corresponding harmonic amplitude increases exponentially with time until nonlinearities can no longer be disregarded. This instability will result first in a deformed (more or less regular) surface and during the initial stages the amplitude grows quite rapidly.
Interesting nonlinear dynamics appears later and periodic steady-state solutions play the major role, because relevant informations can be drawn from their stability

The general idea used here is that if coarsening takes place, this means that every steady-state solution is unstable with respect to wavelength fluctuations and therefore the relevant variable to describe this phenomenon is the wavelength, or, more precisely, the phase of the pattern, since in nonlinear systems it is known that the phase is a more appropriate variable to deal with rather than the wavelength itself [11]. This idea was applied with success to study one dimensional fronts in Ref. [4], where the ability of the system to develop coarsening was directly related to steady-state properties, with no need to perform a forward time-dependent calculation. It will even be shown for several examples below, that the stability or instability of the pattern against phase fluctuations can be concluded analytically. Even more importantly, our approach provides the values of exponents $n$ and $\beta$.

In order to study stability of the periodic steady-state $h_{0}$, we seek for solutions of the nonlinear equation in the form (with $\varepsilon$ small parameter)

$$
h=\tilde{h}_{0}+\varepsilon \tilde{h}_{1}+\ldots,
$$

and linearize the equation. However, in addition to this quite standard study of linear stability, the crux of our method is to introduce a multiscale analysis that will allow us to extract the phase evolution equation, the analysis of which will inform us on the presence of coarsening or the lack thereof. Therefore, besides the fast variables $\mathbf{x}$ and $t$, we introduce slow variables defined as

$$
\mathbf{X}=\varepsilon \mathbf{x}, \quad T=\varepsilon^{2} t .
$$

The perturbation parameter $\varepsilon$ is a small quantity that defines the fact that we are looking for long wavelength modulation of the pattern, which are the most "dangerous"modes (see [4] for more details). In a multiscale spirit fast and slow variables are treated as if they were independent [12]. As already said, it is convenient to work with the phase variables rather than with the spatial variables. For that purpose, we introduce (in two dimensions) two scalar phase variables $\varphi_{1}$ and $\varphi_{2}$. If the pattern is perfectly periodic then these variables are simply given by

$$
\varphi_{1}:=\mathbf{q}_{1} \cdot \mathbf{x}, \quad \varphi_{2}:=\mathbf{q}_{2} \cdot \mathbf{x}
$$

where $\mathbf{q}_{i}=\nabla \varphi_{i}$ are the basis wave vectors defining the symmetry of the stationary periodic pattern. To account for perturbations of the periodic lattice, q-vectors are not just constants but have a dependance on slow scales: $\mathbf{q}=\mathbf{q}(T, \mathbf{X})$; therefore we introduce for convenience the slow phase scales: $\psi_{i}=\varepsilon \varphi_{i}$, so that $\mathbf{q}_{i}=\nabla_{\mathbf{X}} \psi_{i}$ can be expressed as function of slow variables only.

According to this approach, various differential operators in the model equation have to be substituted as 
follows:

$$
\begin{aligned}
\partial_{t} \rightarrow \varepsilon^{2} \partial_{T} & =\varepsilon^{2}\left[\left(\partial_{T} \varphi_{1}\right) \partial_{\varphi_{1}}+\left(\partial_{T} \varphi_{2}\right) \partial_{\varphi_{2}}\right] \\
& =\varepsilon\left[\left(\partial_{T} \psi_{1}\right) \partial_{\varphi_{1}}+\left(\partial_{T} \psi_{2}\right) \partial_{\varphi_{2}}\right]
\end{aligned}
$$

with $\nabla_{0}=\mathbf{q}_{1} \partial_{\varphi_{1}}+\mathbf{q}_{2} \partial_{\varphi_{2}}$ and $\nabla_{\mathbf{X}}=\left(\partial_{X}, \partial_{Y}\right)$. Then expansions (7), (10) and (11) are reported into the model equation (3) which yields (by keeping only terms up to order $\varepsilon$, see Appendix A)

$$
\begin{aligned}
& \varepsilon\left[\left(\partial_{T} \psi_{1}\right) \partial_{\varphi_{1}} \tilde{h}_{0}+\left(\partial_{T} \psi_{2}\right) \partial_{\varphi_{2}} \tilde{h}_{0}\right]= \\
& =-\left(\nabla_{0}+\varepsilon \nabla_{\mathbf{X}}\right) \cdot\left\{\mathbf{j}\left(\nabla_{0} \tilde{h}_{0}\right)+\varepsilon \mathcal{J}\left(\nabla_{0} \tilde{h}_{1}+\nabla_{\mathbf{X}} \tilde{h}_{0}\right)\right. \\
& \left.+\nabla_{0}\left(\nabla_{0}^{2} \tilde{h}_{0}\right)+\varepsilon\left[\nabla_{\mathbf{X}}\left(\nabla_{0}^{2} \tilde{h}_{0}\right)+\nabla_{0}\left(\nabla_{1}^{2} \tilde{h}_{0}+\nabla_{0}^{2} \tilde{h}_{1}\right)\right]\right\}
\end{aligned}
$$

to be studied order by order.

Zeroth order - The zeroth-order defines stationary solutions $\tilde{h}_{0}$ as the unperturbed ones:

$$
0=\nabla_{0} \cdot\left[\mathbf{j}\left(\nabla_{0} \tilde{h}_{0}\right)+\nabla_{0}\left(\nabla_{0}^{2} \tilde{h}_{0}\right)\right]=\nabla_{0} \cdot\left(\mathbf{J}_{0}\right)_{t o t} \equiv \mathcal{N}\left[\tilde{h}_{0}\right],
$$

where $\mathcal{N}$ is a nonlinear operator acting on $\tilde{h}_{0}$. Explicit solutions $\tilde{h}_{0}$ are in general not available, the only basic information being that $\tilde{h}_{0}$ enjoys periodicity properties in $\varphi_{1}, \varphi_{2}$. Focusing our analysis on high symmetry substrates, for which $\langle h\rangle=0$, a stronger condition can be imposed:

$$
\left(\mathbf{J}_{0}\right)_{t o t}=0 \text {. }
$$

First order - At first order we obtain a linear and inhomogeneous equation for $\tilde{h}_{1}$ :

$$
\mathcal{L}\left[\tilde{h}_{1}\right]=g\left(\tilde{h}_{0}, \psi_{1}, \psi_{2}\right)
$$

where

$$
\begin{aligned}
g & \equiv\left(\partial_{T} \psi_{1}\right) \partial_{\varphi_{1}} \tilde{h}_{0}+\left(\partial_{T} \psi_{2}\right) \partial_{\varphi_{2}} \tilde{h}_{0}+\nabla_{0} \cdot\left[\mathcal{J}\left(\nabla_{\mathbf{X}} \tilde{h}_{0}\right)\right. \\
& \left.+\nabla_{\mathbf{X}}\left(\nabla_{0}^{2} \tilde{h}_{0}\right)+\nabla_{0}\left(\nabla_{1}^{2} \tilde{h}_{0}\right)\right]
\end{aligned}
$$

is a function of stationary solutions $\tilde{h}_{0}$, while

$$
\mathcal{L}\left[\tilde{h}_{1}\right] \equiv-\nabla_{0} \cdot\left[\mathcal{J}\left(\nabla_{0} \tilde{h}_{1}\right)+\nabla_{0}\left(\nabla_{0}^{2} \tilde{h}_{1}\right)\right]
$$

is the Fréchet derivative of $\mathcal{N}$, defined as

$$
\mathcal{N}\left[\tilde{h}_{0}+\varepsilon \tilde{h}_{1}\right]=\mathcal{N}_{0}\left[\tilde{h}_{0}\right]+\varepsilon \mathcal{L}\left[\tilde{h}_{1}\right] .
$$

By virtue of translational invariance of $\mathcal{N}$ with respect to space variables, it follows that $\mathcal{N}\left[\tilde{h}_{0}\left(\varphi_{i}+\Delta_{i}\right)\right]$ must vanish as well. In the limit $\Delta_{i} \rightarrow 0$ we get

$$
\mathcal{N}\left[\tilde{h}_{0}\left(\varphi_{i}+\Delta_{i}\right)\right]=\mathcal{N}\left[\tilde{h}_{0}\left(\varphi_{i}\right)\right]+\Delta_{i} \mathcal{L}\left[\partial_{\varphi_{i}} \tilde{h}_{0}\left(\varphi_{i}\right)\right]=0,
$$

which also implies that $\mathcal{L}\left[\partial_{\varphi} \tilde{h}_{0}(\varphi)\right]=0$. Therefore, since $\mathcal{L}\left[\tilde{h}_{1}\right]=0$ has nontrivial solutions $\left(\partial_{\varphi} \tilde{h}_{0}\right)$, the Fredholm alternative theorem [13] can be used for Eq.(15). Such theorem guarantees solutions for Eq. (15) if and only if the so called solvability conditions (expressing the fact that the right hand side of Eq. (15) is orthogonal to the kernel of the adjoint operator of $\mathcal{L}$ ) are verified. These conditions have the following form [14]:

$$
\left\langle v_{i}, g\right\rangle=0
$$

where functions $v_{1}, v_{2}$ verify $\mathcal{L}^{\dagger}[v]=0$. We therefore calculate the adjoint $\mathcal{L}^{\dagger}$ of our linear operator from the definition $\left\langle\mathcal{L}^{\dagger} v, u\right\rangle=\langle v, \mathcal{L} u\rangle$. Given that

$$
\mathcal{L}[u]=-\nabla_{0} \cdot \mathcal{J} \nabla_{0} u-\nabla_{0}^{2}\left(\nabla_{0}^{2} u\right),
$$

$\mathcal{L}$ is self-adjoint if and only if the Jacobian matrix $\mathcal{J}$ is symmetric (see Appendix B). This latter case is definitely the most common one, since we find that it is assured by all the explicit forms of $\mathbf{j}$ used in the literature. We also stress that a symmetric $\mathcal{J}$ means that the current derives from a potential, $\mathbf{J}_{t o t}=-\delta \mathcal{F} / \delta \mathbf{m}$ (see SectionVII for further details).

For the sake of completeness, we must keep in mind that the symmetry property for $\mathcal{J}$ is not a limit in applicability of the current method: a phase diffusion equation could be derived formally without having a linear self-adjoint operator. However, for non adjoint operators, the solutions of $\mathcal{L}^{\dagger}[v]=0$ can be obtained, in general, only numerically [15], even if examples to get them analytically in $1 d$ do exist [4].

According to the above discussion, if $\mathcal{J}$ is symmetric than $\mathcal{L}=\mathcal{L}^{\dagger}$ and $v_{i}=\partial_{\varphi_{i}} \tilde{h}_{0}\left(\varphi_{i}\right)$. It is now possible to rewrite $g$, see Eq. (16), as follows (see Appendix C for more details):

$$
g \equiv\left(\partial_{T} \psi_{1}\right) \partial_{1} h_{0}+\left(\partial_{T} \psi_{2}\right) \partial_{2} h_{0}-\left(\psi_{\alpha}\right)_{\beta \gamma} c_{\beta \gamma}^{\alpha}
$$

where $\partial_{\alpha} \equiv \partial_{\varphi_{\alpha}}$ and $h_{0} \equiv \tilde{h}_{0}$ for ease of notation, and

$$
\begin{aligned}
-c_{\beta \gamma}^{\alpha} & =q_{\delta \nu} \partial_{\delta}\left[\mathcal{J}_{\nu \gamma} \frac{\partial \tilde{h}_{0}}{\partial q_{\alpha \beta}}\right]+2 q_{j \gamma} q_{l \beta} \partial_{\alpha} \partial_{l} \partial_{j} \tilde{h}_{0} \\
& +3 \nabla_{0}^{2} q_{\nu \beta} \partial_{\nu} \frac{\partial \tilde{h}_{0}}{\partial q_{\alpha \gamma}}+\delta_{\beta \gamma} \nabla_{0}^{2} \partial_{\alpha} \tilde{h}_{0},
\end{aligned}
$$

with $q_{i j}=\left(\mathbf{q}_{i}\right)_{j}$ as the $j$-th component of the $i$-th wavevector. Moreover, the compact notation $h_{j} \equiv \partial_{\varphi_{j}} \tilde{h}_{0}$ will be adopted from now on.

By using the above expression for $g$ in the two solvability conditions (20) we obtain the phase diffusion equations $(i=1,2)$ :

$$
\partial_{T} \psi_{i}=\frac{\partial \psi_{\alpha}}{\partial X_{\beta} \partial X_{\gamma}} \tilde{D}_{\beta \gamma}^{i \alpha}, \quad \alpha, \beta, \gamma=1,2
$$

where repeated indices are to be summed over according to Einstein's convention. The diffusion coefficients have the following expressions:

$$
\tilde{D}_{\beta \gamma}^{1 \alpha}=\left[\frac{\left\langle h_{1}, c_{\beta \gamma}^{\alpha}\right\rangle\left\langle h_{2}, h_{2}\right\rangle-\left\langle h_{2}, c_{\beta \gamma}^{\alpha}\right\rangle\left\langle h_{1}, h_{2}\right\rangle}{\left\langle h_{1}, h_{1}\right\rangle\left\langle h_{2}, h_{2}\right\rangle-\left\langle h_{1}, h_{2}\right\rangle^{2}}\right]
$$




\begin{tabular}{c|c|c|c|c} 
symmetry of $h_{0}(\mathbf{x})$ & $\Theta$ & $p$ & invariances & $D_{\beta \gamma}^{i \alpha}$ \\
\hline oblique & no specific & no specific & 2-fold & 12 \\
rhombic & no specific & 1 & 2-fold, $\Pi_{2}$ & 6 \\
rectangular & $\pi / 2$ & no specific & 2-fold, $\Pi_{2}$ & 6 \\
square & $\pi / 2$ & 1 & 4-fold, $\Pi_{2}$ & 3 \\
hexagonal & $\pi / 3$ & 1 & 6-fold, $\Pi_{2}$ & 2 \\
\hline triangular & $2 \pi / 3$ & 1 & 3-fold, $\Pi_{1}$ & 2
\end{tabular}

TABLE I. Presentation of the 5 two dimensional Bravais lattices, classified according to relative orientations between the two q-vectors $(\Theta)$ and their relative amplitude $(p)$; moreover, parity symmetry $\left(\Pi_{2}\right.$ with respect to both space variables, $\Pi_{1}$ with respect to a single space variable) and rotational invariances are specified. The last, extra, row refers to the 3 -fold case. An increasing symmetry corresponds to a decreasing number of independent diffusion coefficients $D_{\beta \gamma}^{i \alpha}$.

and $\tilde{D}_{\beta \gamma}^{2 \alpha} \stackrel{1 \leftrightarrow 2}{=} \tilde{D}_{\beta \gamma}^{1 \alpha}$. It is convenient to define new diffusion coefficients $D_{\beta \gamma}^{i \alpha}$, by regrouping similar derivatives:

$$
D_{\beta \gamma}^{i \alpha}=\left\{\begin{array}{cc}
\tilde{D}_{\beta \gamma}^{i \alpha} & \beta=\gamma \\
\tilde{D}_{\beta \gamma}^{i \alpha}+\tilde{D}_{\gamma \beta}^{i \alpha} & \beta \neq \gamma
\end{array}\right.
$$

Therefore, in the most general case, Eqs. (24) have twelve independent diffusion coefficients. Their expressions are in general quite involved except if some symmetry properties of the steady-state solutions $h_{0}$ are evoked. Symmetry properties will lower the number of independent diffusion coefficients. It should be remembered that $h_{0}$ is a perfectly periodic in-plane pattern, defined by one of the five known two-dimensional Bravais lattices. Selecting one of these patterns for the stationary solution $h_{0}$ means fixing the two q-vectors and the space group symmetry that leave $h_{0}$ unchanged. It is convenient to list the Bravais lattices in a sort of hierarchy to face at once how the demand of symmetry simplifies the expression of diffusion equations. Let us define $\Theta$ as the angle between the two q-vectors and $p$, the proportionality between their moduli:

$$
\cos \Theta:=\frac{\mathbf{q}_{1} \cdot \mathbf{q}_{2}}{\left|\mathbf{q}_{1}\right|\left|\mathbf{q}_{2}\right|}, \quad p:=\frac{\left|\mathbf{q}_{1}\right|}{\left|\mathbf{q}_{2}\right|} .
$$

The proposed order for the five Bravais lattices is shown in Table I. In addition, given its considerable relevance to experiments [16] we also studied the 3 -fold case, that is not included among the Bravais lattices but, nevertheless, can be dealt with using the same method as for the other symmetries. The 3 -fold case is characterized by $\Theta=$ $2 \pi / 3$ and a $p=1$, while the parity symmetry holds for a single space variable only.

In Appendix D we provide an explicit treatment of the phase diffusion equation for the hexagonal symmetry and determine the number of independent coefficients. This serves as a guide for the other symmetries for which we do not report the details. Our results are summarized in Table I: last column reports the number of independent coefficients $D_{\beta \gamma}^{i \alpha}$ corresponding to each pattern symmetry. For oblique, that is the most general one, the number of independent $D_{\beta \gamma}^{i \alpha} \mathrm{s}$ is in fact twelve; for rhombic and rectangular ones parity allows to reduce this number to six; then, the increased degree in the rotational invariance for the square and hexagonal cases implies further reduction to, respectively, three and two independent coefficients. The 3-fold symmetry shares similarities with the hexagonal pattern (albeit the two symmetries are distinct). It turns out that these two symmetries obey formally the same diffusion equation, with the same number of independent coefficients.

In the next Section we are going to exploit the phase diffusion equations for some symmetries and we will report on some far-reaching consequences. In particular, we will examine stability of Eq.(24) with respect to phase perturbations, a relevant information regarding the coarsening problem.

\section{THE COARSENING CONDITIONS}

A coarsening dynamics is signaled by phase instability, i.e. by a phase which increases exponentially with time [17]. Phase diffusion equations, Eqs. (24), are linear and can be solved assuming

$$
\psi_{1,2}(\mathbf{X}, T)=\psi_{1,2}^{(0)} \exp (\Omega T) \exp (i \mathbf{K} \cdot \mathbf{X})
$$

and imposing a null determinant for the linear system with unknowns $\psi_{1,2}^{(0)}$. This way, we can write down a quadratic equation for $\Omega$

$$
\Omega^{2}+f\left(D_{\beta \gamma}^{i \alpha}, \mathbf{K}\right) \Omega+g\left(D_{\beta \gamma}^{i \alpha}, \mathbf{K}\right)=0
$$

and obtain two entire spectra, $\Omega_{1,2}=\Omega_{1,2}(\mathbf{K})$, whose properties depend on the symmetry of $h_{0}$. We present here below detailed results regarding rectangular, square, hexagonal and triangular symmetries. Appendix E lists the $\mathbf{q}$-vectors used in these specific cases. The oblique and rhombic symmetries will not be treated here since they involve quite lengthy expressions. Since we do not expect any new specificity associated with them (see later discussion), we did not feel it worthwhile to dwell on this issue.

\section{A. The hexagonal and triangular symmetries}

In the 6 -fold and in the 3 -fold cases the spectrum turns out to be isotropic in $\mathbf{K}$ and the two eigenvalues are found to be:

$$
\Omega_{1}(K)=-D_{22} K^{2}, \quad \Omega_{2}(K)=-D_{11} K^{2},
$$

where $D_{11}^{11} \equiv D_{22}^{22} \equiv D_{11}$ and $D_{22}^{11} \equiv D_{11}^{22} \equiv D_{22}$ (see Appendix D). Since $D_{22}$ is positive:

$$
D_{22}=\frac{9 q^{2}}{\left\langle h_{\varphi}^{2}\right\rangle}\left\langle h_{12}^{2}\right\rangle>0,
$$


the eigenvalue $\Omega_{1}$ is negative, signaling stability of the pattern. The other eigenvalue, instead, has no a priori fixed sign:

$$
D_{11}=\frac{4 q^{7 / 4}}{\left\langle h_{1}^{2}\right\rangle} \partial_{q}\left(q^{5 / 4}\left\langle h_{12}^{2}\right\rangle\right)
$$

A negative $D_{11}$ would signal instability. We will see later how to determine this sign analytically and how to discriminate among different dynamical scenarios.

\section{B. Square and rectangular symmetries}

For these symmetries, the spectrum of eigenvalues is anisotropic and its analysis is, in principle, more complicated. Let us first consider the square case spectrum:

$$
\Omega_{1,2}(K, \theta)=-\frac{K^{2}}{2}\left[\left(D_{11}+D_{22}\right) \pm \sqrt{\left(D_{11}-D_{22}\right)^{2}+4\left[D_{12}^{2}-\left(D_{11}-D_{22}\right)^{2}\right] \sin ^{2}(\theta)+4\left[\left(D_{11}-D_{22}\right)^{2}-D_{12}^{2}\right] \sin ^{4}(\theta)}\right],
$$

where $K_{1}=K \cos \theta, K_{2}=K \sin \theta$ and where we have used the compact notations: $D_{11}^{11} \equiv D_{22}^{22} \equiv D_{11}, D_{22}^{11} \equiv D_{11}^{22} \equiv$ $D_{22}$, and $D_{12}^{12} \equiv D_{12}^{21} \equiv D_{12}$. Expression (33) shows that extremal values for $\Omega_{1,2}(K, \theta)$ in the $\left(K_{1}, K_{2}\right)$ plane are along the directions $\theta=n \frac{\pi}{2}$ and $\theta=\frac{\pi}{4}+n \frac{\pi}{2}$. Since we are dealing with a 4-fold symmetry, we consider just two cases, for each of which we distinguish two different eigenvalues:

- $\theta=0, \Omega_{1}^{0}(K)=-D_{22} K^{2}, \Omega_{2}^{0}(K)=-D_{11} K^{2}$;

- $\theta=\pi / 4, \Omega_{1}^{\pi / 4}(K)=-\left(D_{11}+D_{22}-D_{12}\right) K^{2} / 2, \Omega_{2}^{\pi / 4}(K)=-\left(D_{11}+D_{22}+D_{12}\right) K^{2} / 2$.

As already seen for hexagonal and triangular symmetries, also in this case one eigenvalue for each couple is always negative, since:

$$
\begin{gathered}
D_{22}=\frac{q^{2}}{\left\langle h_{\varphi}^{2}\right\rangle}\left[\left\langle h_{11}^{2}\right\rangle+3\left\langle h_{12}^{2}\right\rangle\right]>0, \\
D_{11}+D_{22}-D_{12}=\frac{4 q^{2}}{\left\langle h_{\varphi}^{2}\right\rangle}\left\langle h_{11}^{2}\right\rangle>0,
\end{gathered}
$$

while the sign of the other eigenvalues is not obvious, being determined by that of the following expressions:

$$
\begin{gathered}
D_{11}=\frac{1}{\left\langle h_{1}^{2}\right\rangle}\left[\partial_{q}\left(q^{3}\left\langle h_{11}^{2}\right\rangle\right)+q^{3} \partial_{q}\left\langle h_{12}^{2}\right\rangle+q^{2}\left\langle h_{12}^{2}\right\rangle\right], \\
D_{11}+D_{22}+D_{12}=\frac{4}{\left\langle h_{1}^{2}\right\rangle}\left[\frac{1}{2} q^{3} \partial_{q}\left\langle h_{11}^{2}\right\rangle+q^{2}\left\langle h_{11}^{2}\right\rangle+\frac{1}{2} q^{3} \partial_{q}\left\langle h_{12}^{2}\right\rangle+2 q^{2}\left\langle h_{12}^{2}\right\rangle\right] .
\end{gathered}
$$

In Section V we propose calculations of the diffusion coefficients valid in the weakly nonlinear regime, in order to treat those eigenvalues whose sign has not been easily recognizable.

Analogously, for the rectangular case the spectrum takes the following form:

$$
\begin{aligned}
& \Omega_{1,2}(K, s)=-\frac{K^{2}}{2}\left\{\left[\left(D_{11}^{11}+D_{11}^{22}\right)+\left(D_{22}^{11}+D_{22}^{22}-D_{11}^{11}-D_{11}^{22}\right) s\right]\right. \\
& \pm \sqrt{\left.\left(D_{11}^{11}-D_{11}^{22}\right)^{2}+\left[2\left(D_{11}^{11}-D_{11}^{22}\right)\left(D_{22}^{11}-D_{22}^{22}-D_{11}^{11}+D_{11}^{22}\right)+4 D_{12}^{12} D_{12}^{21}\right] s+\left[\left(D_{22}^{11}-D_{22}^{22}-D_{11}^{11}+D_{11}^{22}\right)^{2}-4 D_{12}^{12} D_{12}^{21}\right] s^{2}\right\}}
\end{aligned}
$$

where $s \equiv \sin ^{2}(\theta)$. The extremal values are now obtained not only along the maximal symmetry directions, namely along $\theta=n \pi$ and $\theta=n \pi \pm \pi / 2$, but also along two new other directions we are able to specify in the weakly nonlinear regime (see Appendix F). According to such approximation, corresponding to steady states of small 
amplitude $a$, these directions are close to $\theta=\pi / 4$ :

$$
\sin ^{2}(\theta)=\frac{1}{2} \pm 2 \sqrt{2} \frac{q^{4}}{m^{2}} \frac{p^{2}-1}{\left(p^{2}+1\right)^{2}} p \equiv \frac{1}{2} \pm O\left(a^{2}\right),
$$

where $m=q^{3} \partial_{q}\left(a^{2}\right) / a^{2}$ and $p$ is defined by Eq. (27). The eigenvalues corresponding to the two first extremal directions are:

$$
\begin{array}{ll}
\text { - } & \theta=0 \\
& \Omega_{1}^{0}(K)=-D_{11}^{22} K^{2}, \\
& \Omega_{2}^{0}(K)=-D_{11}^{11} K^{2} ; \\
\text { - } & \theta=\pi / 2 \\
& \Omega_{1}^{\pi / 2}(K)=-D_{22}^{11} K^{2}, \\
& \Omega_{2}^{\pi / 2}(K)=-D_{22}^{22} K^{2} .
\end{array}
$$

Again, one eigenvalue for each couple is always negative, since:

$$
\begin{aligned}
& D_{22}^{11}=\frac{q^{2}}{\left\langle h_{1}^{2}\right\rangle}\left[\left\langle h_{11}^{2}\right\rangle+3 p^{2}\left\langle h_{12}^{2}\right\rangle\right]>0, \\
& D_{11}^{22}=\frac{q^{2}}{\left\langle h_{2}^{2}\right\rangle}\left[3\left\langle h_{12}^{2}\right\rangle+p^{2}\left\langle h_{22}^{2}\right\rangle\right]>0
\end{aligned}
$$

while the other has no obvious sign, as it is fixed by that of the following expressions:

$$
\begin{aligned}
& D_{11}^{11}=\frac{1}{\left\langle h_{1}^{2}\right\rangle}\left[\partial_{q}\left(q^{3}\left\langle h_{11}^{2}\right\rangle\right)+q^{3} p^{2} \partial_{q}\left\langle h_{12}^{2}\right\rangle+q^{2} p^{2}\left\langle h_{12}^{2}\right\rangle\right] \\
& D_{22}^{22}=\frac{1}{\left\langle h_{2}^{2}\right\rangle}\left[p^{2} \partial_{q}\left(q^{3}\left\langle h_{22}^{2}\right\rangle\right)+q^{3} \partial_{q}\left\langle h_{12}^{2}\right\rangle+q^{2}\left\langle h_{12}^{2}\right\rangle\right] .
\end{aligned}
$$

It is worth notice that $D_{22}^{11}=D_{11}^{22}$ and $D_{11}^{11}=D_{22}^{22}$, for $p=1[18]$. The other two extremal directions, defined by Eq. (39) have to be considered in the weakly nonlinear regime. The reader can find calculations in Appendix F. Here, it suffices to say that, also in the rectangular case, once the direction has been fixed, the sign of one eigenvalue is negative while the sign of the other is not evident.

As a summary of this section we can highlight two important conclusions. In the hexagonal and triangular symmetries, one eigenvalue is positive (phase instability) if the quantity (see Eq. (32))

$$
\mathcal{A} \equiv q^{5 / 4}\left\langle h_{12}^{2}\right\rangle
$$

is a decreasing function of the wavenumber $q$. The quantity $\mathcal{A}$ depends only on the properties of the steady-state solutions. Thus, determining whether coarsening occurs or not can be decided on the inspection of steady-state solutions only. This result generalizes our previous onedimensional study to two dimensions [4], where we found that coarsening occurs if $\left\langle h_{0}^{2}\right\rangle$ (which is nothing but the amplitude of the pattern) is a decreasing function of $q$. In two dimensions [19] we had previously found for the time-dependent Ginzburg-Landau equation and for the Cahn-Hilliard equation that a certain quantity, different both from that of the one-dimensional problem and from Eq. (44), must be a decreasing function of $q$. Thus, we can state that the nature of the function whose decreasing character determines stability depends on the space dimension and on the class of the considered equations [20].

The second important conclusion is that our results in this section do not depend on the nature of the current $\mathbf{j}$ entering in Eq. (3). The reader can refer to Appendix D in order to check this statement in detail for the 6-fold symmetry.

\section{THE DIFFUSION EQUATION IN THE WEAKLY NONLINEAR REGIME}

In this Section our aim is to analyze if coarsening occurs or not, while the determination of coarsening exponents will be presented in the next section. In order to determine the dynamical scenarios for our growth equation, we need an evaluation of the signs of appropriate diffusion coefficients, see Eqs.(D2,36,37,42). This task can, in general, be performed only numerically by solving for the steady-state solutions. However, by restricting ourselves to a weakly nonlinear analysis, some analytical results can be obtained. To that end we assume that the amplitude of the stationary solution $h_{0}$ is small. We have already performed the general linear analysis of our equation, which has resulted into the spectrum (6). In a weakly nonlinear approach we can push further this stability analysis extracting an approximated solution for $h_{0}$ in power series of the amplitudes of the Fourier modes. Thanks to the periodic character of the stationary solution we can express $h(\mathbf{x}, t)$ with a Fourier series that can be truncated at some order. The small amplitude limit is legitimate as long as $k \rightarrow 1$, so that higher harmonics are stable, ensuring the consistency of the truncation of the series.

Since the symmetry of the growing pattern is identical or lower than substrate symmetry, an isotropic current is the most general one, i.e. compatible with any Bravais lattice. We consider a generic class of isotropic currents $\mathbf{j}\left(\mathbf{m}, c_{2}, c_{4}\right)=\mathbf{m}\left(1+c_{2} \mathbf{m}^{2}+c_{4} \mathbf{m}^{4}\right)$ so that Eq. (3) becomes:

$$
\begin{aligned}
\partial_{t} h & =\tilde{L}[h]-c_{2}\left[3\left(h_{x}^{2} h_{x x}+h_{y}^{2} h_{y y}\right)+h_{y}^{2} h_{x x}+h_{x}^{2} h_{y y}\right. \\
& \left.+4 h_{x} h_{y} h_{x y}\right]-c_{4}\left[5\left(h_{x}^{4} h_{x x}+h_{y}^{4} h_{y y}\right)+h_{x}^{4} h_{y y}+h_{y}^{4} h_{x x}\right. \\
& \left.+6 h_{x}^{2} h_{y}^{2}\left(h_{x x}+h_{y y}\right)+8 h_{x y}\left(h_{x} h_{y}^{3}+h_{x}^{3} h_{y}\right)\right]
\end{aligned}
$$

where $\tilde{L}[h]=-\nabla \cdot(\nabla h)-\nabla^{4} h$ is the linear part. We focus here on the square and hexagonal symmetries for which the determination of the steady-state solution $h_{0}$ is relatively simple.

For a square symmetry, adopting the wave vector directions which are specified in Appendix E, we can write $h(\mathbf{x}, t)=\sum_{n, m} a_{n, m}(t) e^{i q(n x+m y)}$. The constant term $(n=0, m=0)$ is zero because of the condition 
$\langle h\rangle=0$. Given the real character of $h(\mathbf{x}, t)$ and its paritysymmetry with respect to both space variables, it follows that $a_{n, m}^{*}=a_{-n,-m}$ and $a_{-n, m}=a_{n, m}=a_{m,-m}$. Invariance under $\pi / 2$ rotations implies the following additional relations:

$$
h(x, y)=h(-y, x)=h(-x,-y)=h(y,-x),
$$

which translate into the following conditions for the harmonic amplitudes:

$$
a_{n, m}=a_{m,-n}=a_{-n,-m}=a_{-m, n} .
$$

Therefore, at first order $(n, m= \pm 1)$ :

$$
h(\mathbf{x}, t)=a_{1}(t)\left(e^{i q(x+y)}+e^{i q(x-y)}+\text { c.c. }\right),
$$

with $a_{1,1}=a_{-1,1}=a_{-1,-1}=a_{1,-1} \equiv a_{1}$, so the same dynamics equation holds for the four harmonics. This series can be written in the more convenient form $h_{0}=$ $4 a_{1} \cos \varphi_{1} \cos \varphi_{2}$ and diffusion coefficients (36) and (37) can be explicitly calculated in the small amplitude limit:

$$
\begin{aligned}
D_{11} & \simeq \frac{4}{\left\langle h_{\varphi}^{2}\right\rangle}\left[\frac{d}{d q}\left(q^{3} a_{1}^{2}\right)+q^{3} \frac{d}{d q} a_{1}^{2}+q^{2} a_{1}^{2}\right] \\
& \simeq \frac{8 q_{\mathrm{MAx}}^{3}}{\left\langle h_{\varphi}^{2}\right\rangle} \frac{d a_{1}^{2}}{d q}, \\
D_{11} & +D_{22}+D_{12} \simeq \frac{16 q_{\mathrm{MAx}}^{3}}{\left\langle h_{\varphi}^{2}\right\rangle} \frac{d a_{1}^{2}}{d q},
\end{aligned}
$$

with the substitution $q=q_{\mathrm{MAx}}=1 / \sqrt{2}$ in the last passages. It is now evident how the sign of the eigenvalues is directly related to the increasing or decreasing character of the steady amplitude $a_{1}$ as function of the steady wave length $\lambda=2 \pi / q$. Reporting expansion (48) into Eq. (45) we find the equation obeyed by $a_{1}$ :

$$
\dot{a}_{1}=a_{1}\left(\omega_{1}+c_{2} 20 q^{4} a_{1}^{2}+224 c_{4} q^{6} a_{1}^{4}\right),
$$

where $\omega_{1}:=\omega(k=\sqrt{2} q)$, and then solve for stationary solutions:

$$
a_{1}=0, \quad \omega_{1}+20 c_{2} q^{4} a_{1}^{2}+224 c_{4} q^{6} a_{1}^{4}=0 .
$$

We conclude that the number of stationary solutions depends on values of $c_{2}$ and $c_{4}$ : coarsening occurrence is directly associated with specific forms of $\mathbf{j}$ currents, whose expression determines one of three possible scenarios.

Let's first consider the case in which $c_{4}=0$. We find:

$$
a_{1}=\sqrt{\frac{\omega_{1}}{-20 c_{2} q^{4}}}
$$

so that stationary solutions corresponding to the band of wavevectors such that $\omega_{1}>0$ exist only if $c_{2}<0$. Using Eqs. (49), we now obtain:

$$
D_{11}=\frac{8}{5\left\langle h_{\varphi}^{2}\right\rangle} \frac{1}{c_{2}}
$$

Consequently, phase equation eigenvalues are positive, implying instability with respect to phase fluctuations, i.e. coarsening (this also holds for $c_{2}>0$ and $c_{4}<0$ ). Instead, in the case where $c_{2}>0$, we can easily see that eigenvalues are negative, meaning no coarsening at all. Finally, in the case $c_{2}<0$ and $c_{4}>0$, we find interrupted coarsening: the length scale $L$ of the pattern increases until reaching a certain maximum wavelength $\lambda=2 \pi / q$. To fix idea, and without loss of generality, we set $c_{2}=-1$ and deduce the two stationary solutions from Eqs. (52):

$$
x_{ \pm}\left(q, c_{4}\right)=\frac{10 q^{4} \pm \sqrt{\left(10 q^{4}\right)^{2}-224 c_{4} q^{6} \omega_{1}}}{224 c_{4} q^{6}} \equiv a_{1}^{2},
$$

which both coincide at the maximum reachable length scale, where coarsening is interrupted.

For the hexagonal case, we proceed in the same way, setting a new truncated Fourier series:

$$
h(\mathbf{x}, t)=a_{1}(t)\left(e^{i q / 2(x+\sqrt{3} y)}+e^{i q x}+e^{i q / 2(x-\sqrt{3} y)}+\text { c.c. }\right),
$$

with $a_{1,1}=a_{1,0}=a_{0,-1}=a_{-1,-1}=a_{-1,0}=a_{0,1} \equiv a_{1}$, so the same dynamics equation holds for any of the six harmonics [21]. Again, reporting Eq.(56) into Eq.(45), we obtain the amplitude equation:

$$
\dot{a}_{1}=a_{1}\left(\omega_{1}+c_{2} 9 q^{4} a_{1}^{2}+94 c_{4} q^{6} a_{1}^{4}\right),
$$

where $\omega_{1}:=\omega(k=q)$. Because this equation has the same structure and the same signs in front of each term as in Eq. (51), the same conclusions as above are reached, namely we have coarsening, no coarsening and interrupted coarsening scenarios depending on the signs of the coefficients $c_{2}$ and $c_{4}$.

The above results can be extended to other symmetries, including the 3-fold symmetry, which does not correspond to a Bravais lattice. In that case, the starting Fourier series corresponds to a linear combination of $h(\mathbf{x}, t)$ and $h\left(\mathbf{x}-\mathbf{x}_{0}, t\right)$, where $h(\mathbf{x}, t)$ is given by Eq. (56) and $\mathbf{x}_{0}=\left(\frac{4}{3} \pi / q, 0\right)[22]$.

It is worthnoting that in the limit of weak amplitude, the coarsening criterion always corresponds to the requirement that the amplitude of the stationary solution is a decreasing function of the wavevector. This is a trivial consequence of the single harmonic approximation, where $h_{0}(\mathbf{x})=a_{1}(q) \times$ exponential factors.

\section{UNIVERSALITY CLASSES}

In this section our aim is to extract analytically the coarsening exponents $\beta$ and $n$, defined as in Sec. I. In order to determine the exponent we make use of the temporal behavior of the phase, see Eq. (28), whose amplitude increases as $\psi^{(0)} e^{\Omega T}$. The relevant time scale of the phase instability is therefore set by $\Omega^{-1}$. According to Sec. IV, the unstable mode has an eigenvalue of the form $\Omega=-K^{2} \mathcal{D}$, where $\mathcal{D}$ is a suitable combination of diffusion coefficients and whose negative sign indicates 
instability. Therefore, if $L$ is the typical size of mounds after a time $t$, we have $T=\varepsilon^{2} t, K \approx \frac{1}{\varepsilon L}$ and

$$
|\mathcal{D}(q)| \approx \frac{L^{2}}{t},
$$

with $q=2 \pi / L[23]$.

It turns out that the coarsening exponent $n$ only depends on one single property (see below) of the current j entering the general equation (3), while its symmetry, as well as the pattern symmetry, is definitely irrelevant. The only essential ingredient is whether the current leads or not to a slope selection. We find $n=1 / 3$ for $\mathbf{j}$ currents giving rise to mounds that grow with a certain constant slope and $n=1 / 4$ otherwise. Let us show more precisely these results.

Let us consider the square symmetry. We have seen that coarsening occurs if at least one of the expressions given by (36) or (37) is negative. Consider one scalar product entering expression (36):

$$
\left\langle h_{11}^{2}\right\rangle=\frac{1}{(2 \pi)^{2}} \int_{0}^{\lambda} d x \int_{0}^{\lambda} d y \frac{1}{q^{2}}\left(\frac{\partial^{2} h_{0}}{\partial x^{2}}\right)^{2} .
$$

For systems exhibiting slope selection, the current $\mathbf{j}(\mathbf{m})$ has zeros for finite values $\mathbf{m}^{*}$ of the slope, therefore $\mathbf{m}^{*}=$ $\left(\partial_{x} h_{0}, \partial_{y} h_{0}\right)$ is constant everywhere but along domain walls, that have a finite but small thickness. Let's denote the thickness by $\delta$. In the large wavelength limit $\delta \ll \lambda$ we can also assume that inside domain wall there is a linear space dependence for the slope $\mathbf{m}$ : for example, in Eq. (59), $\partial h_{0} / \partial x=m_{x} \approx A x+B y$, with $A$ and $B$ real constants, whose exact values are unimportant for our purposes. Estimation of Eq. (59), therefore, yields

$$
\left\langle h_{11}^{2}\right\rangle \simeq \frac{1}{(2 \pi)^{2}} \frac{1}{q^{2}} \int_{0}^{\lambda} d y \int_{\lambda-\delta}^{\lambda} d x A^{2} \simeq c_{11} \lambda^{3}+o\left(\lambda^{3}\right)
$$

with $c_{11}$ a positive constant. Similar considerations lead to $\left\langle h_{12}^{2}\right\rangle \simeq c_{12} \lambda^{3}$ and $\left\langle h_{1,2}^{2}\right\rangle \simeq c_{\varphi} \lambda^{2}$, where constants, again, are positive. We straightforwardly obtain $D_{11}=-2 q c_{12} / c_{\varphi}$ from Eq. (36) and $\left(D_{11}+D_{22}+D_{12}\right)=$ $2 q\left(c_{12}-c_{11}\right) / c_{\varphi}$, with $c_{11} \geq c_{12}$ [24], from Eq. (37): both coefficients are evidently negative[25], and they have the same $q$-dependence. Using (58) the coarsening exponent can be easily extracted:

$$
L \sim t^{1 / 3} .
$$

For models without slope selection, the current $\mathbf{j}$ has no zeros. A prototype of this kind of currents is asymptotically represented by $\mathbf{j}(\mathbf{m}) \simeq 1 /|\mathbf{m}|^{\alpha}, \alpha>1$. Exploiting Eq. (14) and coupling it with the asympotic expression of the current, we obtain

$$
-\nabla^{2} \mathbf{m} \simeq 1 /|\mathbf{m}|^{\alpha} .
$$

We now switch to polar coordinates and make the assumption that mound profile changes only along one

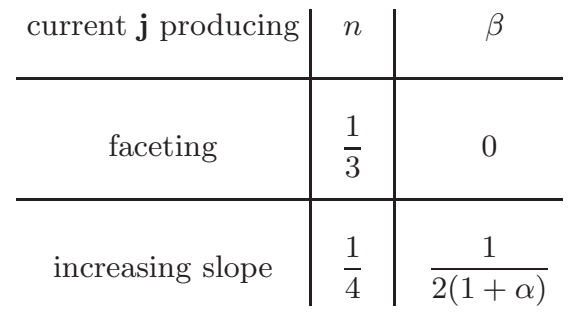

TABLE II. The value of the coarsening exponent $n$ and of the exponent $\beta\left(|\mathbf{m}(t)| \approx t^{\beta}\right.$ for large $t$, for the two universality classes resulting from our study. The exponent $\alpha$ appears in the relation $\mathbf{j}(\mathbf{m}) \approx 1 /|\mathbf{m}|^{\alpha}$ for large $\mathbf{m}$.

direction but remains constant along the perpendicular one, so that Eq.(62) can be mapped onto a onedimensional equation: $m^{\prime \prime}+(1 / r) m^{\prime}+1 / m^{\alpha}$, neglecting the angular dependence for $\mathbf{m}$. Plugging in it a solution of the form $m \simeq A r^{\gamma}$, we find $\gamma=2 /(\alpha+1)$ and finally, calculating again the scalar products in Eqs. (36) and (37) for the square symmetry case, we get

$$
L \sim t^{1 / 4}
$$

for any value of $\alpha$.

We can gain further insight from dimensional considerations: if $m \approx L^{2 / \alpha+1}$, then $m \approx t^{\beta}=t^{2 n / \alpha+1}$, therefore giving $\beta=1 /(2(\alpha+1))$. We have found that all the other symmetries hereby mentioned produce exactly the same exponents, $n$ and $\beta$, pointing to the existence of universality classes. Our results are summarized in Table II.

It is worth comparing our results for $n$ with the corresponding values for the one-dimensional growth models. The models with constantly increasing slope yield $n=1 / 4[4,26]$, as we have also found in two dimensions. The $1 d$ model with faceting, instead, is known to produce a logarithmic coarsening in the absence of noise $[4,27]$ and $n=1 / 3$ when noise is present [28]. We conclude, with the caveat of noise and in analogy with the case of phase separation processes (see Sec.VII.B for a thorough discussion), that the dimension of physical space, $d$, seems [29] to be irrelevant for our class of growth models, Eq. (3).

\section{DISCUSSION}

\section{A. The context}

The field of crystal growth processes by a vapour phase has been very active in the past twenty-five years, involving experiments, simulations, and analytic studies. General references of special interest for the present article are three review papers $[6,30,31]$ and one book [32].

Rigorous results for model equations included in the class (3) studied here are very rare. We are aware of two exact inequalities, concerning isotropic currents and which are in agreement with our universality classes: Kohn\&Yan [33] studied the faceted case, finding $n \leq 1 / 3$; 
Bo\&Li [34] studied the increasing slope case, finding $n \leq 1 / 4$. Another worth mentioning paper is the study by Watson\&Norris [35] of the faceted case with a threefold symmetric current: authors find $n=1 / 3$, also in agreement with our findings.

Less rigorous results are often based on approximate evaluations of the different terms appearing in the equation and on how such terms depend on scale length $L$. A significant amount of effort has been devoted to such an approach by Golubović and collaborators [31]. While in the "no faceted" case [36] results are in agreement with ours, the faceted case is controversial. More precisely, in Ref. [37] the six-fold symmetric case gives $n=1 / 3$, while in Ref. [38] the four-fold case may give either $n=1 / 3$ or $n=1 / 4$, depending on the details of the current. Siegert too, already a few years before, had claimed to find a slower coarsening, $n=1 / 4$, when integrating numerically a (3)-like equation with square symmetry and faceted morphology [39].

The peculiar result $n=1 / 4$ for square symmetry would be related, according to the above mentioned authors, to the existence of two different types of domain walls, pyramid edges and roof tops: in the former case only one component of the slope changes, while in the latter case both components change. Roof tops, which are not present in a regular, periodic square lattice, act as dislocations and would play a major role in their simulations, slowing down coarsening. However, they also claim that the system would be frozen in the absence of roof tops, because the square pattern would be metastable. The last statement is in contrast to our findings, according to which the square pattern is expected to be linearly unstable. We think that the square case would require some more analysis to gain more insights on the role of defects, as well as on the roles of the specific form of the current, of the initial conditions and of the simulation time. For the sake of completeness we also make our reader aware that the same considerations just expressed for the square case might be extended to the less studied rectangular case, since a different coarsening exponent can be found in the literature $(n=2 / 7)$ [31]. Again, also for this symmetry, coarsening is bound to the presence of dislocations and the specific form of the current might be relevant (e.g., its derivability from a potential).

While comparing with previous studies of the same class of equations is straightforward, comparing with Kinetic Monte Carlo simulations and with experiments becomes difficult and dangerous, mainly for two reasons: (i) Is the system (the simulated system or the real system) described by a (3)-like equation or different equations are more appropriate? (ii) Does the simulation or the experiment attain large enough times to probe the asymptotic regime? Honestly, the variety of results of simulations/experiments and the above two questions prevent from giving a clear picture of such results and from connecting them to specific model equations. As for experiments, we refer the reader to Table 2 or Ref. [6] and to Table 4.2 of Ref. [32]. We close this Section by giving a few more details on point (i), here above.

Equation (3) is a class of general models because $\mathbf{j}(\mathbf{m})$ has the only requirement to be linear at small slopes. Such equation cannot cover any possible model of growth by vapor deposition. For example, some studies have suggested a higher order linear term, which would produce slower coarsening [36] (if two linear terms of different order are present, crossover effects are expected, depending on their relative strength). A second remark is related to the up-down symmetry of the emerging morphology. Eq. (3) is invariant under the transformation $h \rightarrow-h$ but such a symmetry is weak or absent in simulations and experiments. Therefore, symmetry breaking terms have been introduced, for example in Ref. [40]. While in $d=1$ such a term seems to be irrelevant [41], the question of its relevance in $d=2$ is still open. A final comment concerns the form of $\mathbf{j}(\mathbf{m})$. Whatever its symmetry, we have assumed it is analytical at $\mathbf{m}=0$. However, some results [42] suggest that step-edge diffusion might produce a current which is singular at vanishing slope.

\section{B. Crystal growth vs phase separation}

In the previous Section we have discussed how our results compare with other studies on crystal growth. Here we rather focus on differences and similarities with phase separation processes.

First of all, the irrelevance of space dimensionality we have found to be valid for our growth model Eq.(3) is also a well-known feature of phase-separation processes, as long as $T_{c}>0$. In such context, for a scalar order parameter the coarsening exponents are $n=1 / 2$ for a nonconserved order parameter and $n=1 / 3$ for a conserved one $[2,4]$. However, in $d=1$, such figures are found when noise is present [28], otherwise a slow logarithmic coarsening appears [27]. The one dimensional growth model with faceting is equivalent to a conserved phase separation process (so called model B of dynamics or Cahn- Hilliard equation). Therefore, it is not surprising that it gives logarithmic coarsening without noise and $n=1 / 3$ in the presence of noise. However, the analogy with phase separation processes cannot be pushed further, because our growth model has, in $2 d$, peculiar proprieties.

Even if our multiscale approach is applicable, in principle, to any nonequilibrium current $\mathbf{j}(\mathbf{m})$, we have considered here the case of symmetric Jacobian matrix $\mathcal{J}$, which means $\partial j_{i} / \partial m_{\ell}=\partial j_{\ell} / \partial m_{i}$. This condition is satisfied by all crystal growth equations we are aware of and it is equivalent to saying that dynamics can be cast into a variational formulation. In fact, Eq. (3) can be rewritten as:

$$
\frac{\partial h}{\partial t}=\nabla \cdot \frac{\delta \mathcal{F}}{\delta \mathbf{m}}
$$


if and only if $\mathcal{J}$ is symmetric, where

$$
\mathcal{F}=\int d \mathbf{x}\left\{\frac{1}{2}\left[\left(\nabla m_{x}\right)^{2}+\left(\nabla m_{y}\right)^{2}\right]+V(\mathbf{m})\right\},
$$

so that

$$
\begin{aligned}
\frac{d \mathcal{F}}{d t} & =\int d \mathbf{x} \frac{\delta \mathcal{F}}{\delta m_{i}} \frac{\partial m_{i}}{\partial t}=\int d \mathbf{x} \frac{\delta \mathcal{F}}{\delta m_{i}} \frac{\partial}{\partial x_{i}}\left(\nabla \cdot \frac{\delta \mathcal{F}}{\delta \mathbf{m}}\right) \\
& =-\int d \mathbf{x}\left(\nabla \cdot \frac{\delta \mathcal{F}}{\delta \mathbf{m}}\right)^{2} \leq 0 .
\end{aligned}
$$

Taking the gradient of both sides of Eq. (64) we obtain

$$
\frac{\partial \mathbf{m}}{\partial t}=\nabla\left(\nabla \cdot \frac{\delta \mathcal{F}}{\delta \mathbf{m}}\right)
$$

which is reminiscent of the B-dynamics for a conserved, vector order parameter:

$$
\frac{\partial \mathbf{m}}{\partial t}=\nabla^{2}\left(\frac{\delta \mathcal{F}}{\delta \mathbf{m}}\right)
$$

The very first remark is that we can evoke some similarity with a phase separation process only in the case of faceting. In fact, phase separation requires that $V(\mathbf{m})$ has minima for finite $\mathbf{m}$ and these minima correspond to the magic slopes for which $\mathbf{j}$ vanishes and which keep constant in time during the coarsening process. The second remark is that Eqs. (67) and (68) are different (see what $\nabla$ applies to) and the order parameter $\mathbf{m}$ is also different: in Eq. (67) $\mathbf{m}=\nabla h$, which means $\nabla \times \mathbf{m}=0$. In simple terms, $\mathbf{m}=\nabla h$ implies that domain walls must be straight lines, because they result from the intersection of two planes (regions of constant $\mathbf{m}$ ), while the shape of domain walls in standard phase separation processes has no such constraint.

Because of these differences between Eqs. (67) and (68), it should not appear surprising they give different coarsening exponents. While the crystal growth equation (67) gives $n=\frac{1}{3}$ irrespectively of the symmetry of $V(\mathbf{m})$, for the phase separation process, Eq. (68), we have $n=\frac{1}{4}$ if $V(\mathbf{m})$ has a continuous family of minima (i.e. an infinite number of minima corresponding to a circularly symmetric current), and $n=\frac{1}{3}$ if $V(\mathbf{m})$ has a finite number of minima (the current has rotational symmetry under a specific angle), because such case corresponds to a scalar order parameter $[43,44]$.

In conclusion, we have proposed a classification of important unstable crystal growth dynamics in terms of universality classes, detecting what features are relevant (faceting or not) and what features are irrelevant (symmetry of the pattern, the symmetry of the surface mass current and the space dimensionality). Therefore, we have shown that Eq. (3) has distinct properties and critical exponents, conferring to unstable crystal growth a place as novel nonequilibrium paradigm.

\section{ACKNOWLEDGMENTS}

CM thanks CNR for a Short-Term Mobility award from their International Exchange Program and CNES for financial support.

\section{Appendix A: Growth equation perturbative expansions}

Using (10) and (11), we can express different operators of our growth model equation (3). For the Laplacian we have:

$$
\nabla^{2}=\nabla_{0}^{2}+\varepsilon\left[\nabla_{0} \nabla_{\mathbf{X}}+\nabla_{\mathbf{X}} \nabla_{0}\right] \equiv \nabla_{0}^{2}+\varepsilon \nabla_{1}^{2} .
$$

The expansion of the current takes the form:

$$
\begin{aligned}
\mathbf{j}(\nabla h) & =\mathbf{j}\left(\nabla_{0}\left(\tilde{h}_{0}+\varepsilon \tilde{h}_{1}\right)+\varepsilon \nabla_{\mathbf{X}}\left(\tilde{h}_{0}+\varepsilon \tilde{h}_{1}\right)\right)= \\
& =\mathbf{j}\left(\nabla_{0} \tilde{h}_{0}\right)+\varepsilon \mathcal{J}\left(\nabla_{0} \tilde{h}_{1}+\nabla_{\mathbf{X}} \tilde{h}_{0}\right),
\end{aligned}
$$

with $\mathcal{J}$ being the Jacobian matrix of $\mathbf{j}$, and:

$$
\nabla^{2} h=\left(\nabla_{0}^{2}+\varepsilon \nabla_{1}^{2}\right)\left(\tilde{h}_{0}+\varepsilon \tilde{h}_{1}\right)=\nabla_{0}^{2} \tilde{h}_{0}+\varepsilon\left(\nabla_{1}^{2} \tilde{h}_{0}+\nabla_{0}^{2} \tilde{h}_{1}\right)
$$

with its gradient:

$$
\nabla\left(\nabla^{2} h\right)=\nabla_{0}\left(\nabla_{0}^{2} \tilde{h}_{0}\right)+\varepsilon\left[\nabla_{\mathbf{X}}\left(\nabla_{0}^{2} \tilde{h}_{0}\right)+\nabla_{0}\left(\nabla_{1}^{2} \tilde{h}_{0}+\nabla_{0}^{2} \tilde{h}_{1}\right)\right] .
$$

Thus, our model equation (3) becomes at first order:

$$
\begin{aligned}
& \varepsilon\left[\left(\partial_{T} \psi_{1}\right) \partial_{\varphi_{1}} \tilde{h}_{0}+\left(\partial_{T} \psi_{2}\right) \partial_{\varphi_{2}} \tilde{h}_{0}\right]= \\
& =-\left(\nabla_{0}+\varepsilon \nabla_{\mathbf{X}}\right) \cdot\left\{\mathbf{j}\left(\nabla_{0} \tilde{h}_{0}\right)+\varepsilon \mathcal{J}\left(\nabla_{0} \tilde{h}_{1}+\nabla_{\mathbf{X}} \tilde{h}_{0}\right)\right. \\
& \left.+\nabla_{0}\left(\nabla_{0}^{2} \tilde{h}_{0}\right)+\varepsilon\left[\nabla_{\mathbf{X}}\left(\nabla_{0}^{2} \tilde{h}_{0}\right)+\nabla_{0}\left(\nabla_{1}^{2} \tilde{h}_{0}+\nabla_{0}^{2} \tilde{h}_{1}\right)\right]\right\} .
\end{aligned}
$$

\section{Appendix B: Expression for the adjoint $\mathcal{L}^{\dagger}$}

From the first order contribution (17) of our growth equation we define the linear operator $\mathcal{L}$ :

$$
\mathcal{L}[u]=-\nabla_{0} \cdot \mathcal{J} \nabla_{0} u-\nabla_{0}^{2}\left(\nabla_{0}^{2} u\right)
$$

In order to determine the adjoint operator we split the above linear operator into two parts

$$
\begin{aligned}
\left\langle\mathcal{L}^{\dagger}\right. & v, u\rangle=-\left\langle v, \nabla_{0} \cdot \mathcal{J} \nabla_{0} u\right\rangle \\
\left\langle\mathcal{L}^{\dagger}\right. & \left.{ }_{2} v, u\right\rangle=-\left\langle v, \nabla_{0}^{2}\left(\nabla_{0}^{2} u\right)\right\rangle .
\end{aligned}
$$

and apply the definition $\left\langle\mathcal{L}^{\dagger} v, u\right\rangle=\langle v, \mathcal{L} u\rangle$ for each term separately. Integration by part in the second term yields:

$$
\left\langle\mathcal{L}^{\dagger}{ }_{2} v, u\right\rangle=-\left\langle v, \nabla_{0}^{2}\left(\nabla_{0}^{2} u\right)\right\rangle=-\left\langle\nabla_{0}^{2} v, \nabla_{0}^{2} u\right\rangle=-\left\langle\nabla_{0}^{4} v, u\right\rangle,
$$

(integrals over the surface vanish because of periodicity of the integrand over the interval $[0,2 \pi])$. Thus $\mathcal{L}_{2}$ is selfadjoint. Regarding the first term $\mathcal{L}_{1}$ it is more convenient 
to use explicitly the integral notation for the scalar product and express it in terms of components:

$$
\begin{aligned}
\left\langle\mathcal{L}^{\dagger}{ }_{1} v, u\right\rangle & =-\left\langle v, \nabla_{0} \cdot \mathcal{J} \nabla_{0} u\right\rangle \equiv \\
& \equiv-\frac{1}{(2 \pi)^{2}} \iint v^{*}\left[\left(\nabla_{0}\right)_{i} \mathcal{J}_{i j}\left(\nabla_{0}\right)_{j} u\right]= \\
& =+\frac{1}{(2 \pi)^{2}} \iint\left[\left(\nabla_{0}\right)_{i} v^{*}\right]_{i j}\left[\left(\nabla_{0}\right)_{j} u\right]= \\
& =+\frac{1}{(2 \pi)^{2}} \iint \mathcal{J}_{i j}\left[\left(\nabla_{0}\right)_{i} v^{*}\right]\left[\left(\nabla_{0}\right)_{j} u\right]= \\
& =-\frac{1}{(2 \pi)^{2}} \iint\left[\left(\nabla_{0}\right)_{j} \mathcal{J}_{i j}\left(\nabla_{0}\right)_{i} v^{*}\right] u,
\end{aligned}
$$

i.e.

$\mathcal{L}^{\dagger}{ }_{1}=-\left(\nabla_{0}\right)_{i} \mathcal{J}_{j i}\left(\nabla_{0}\right)_{j}=-\left(\nabla_{0}\right)_{i} \mathcal{J}_{i j}^{T}\left(\nabla_{0}\right)_{j} \equiv-\nabla_{0} \cdot \mathcal{J}^{T} \nabla_{0}$.

Therefore the total $\mathcal{L}$ operator is self-adjoint if and only if the Jacobian matrix of the current $\mathbf{j}$ is symmetric.

\section{Appendix C: Solvability conditions}

Let us write down explicitly the solvability equations (20):

$$
\begin{aligned}
g & \equiv\left(\partial_{T} \psi_{1}\right) h_{1}+\left(\partial_{T} \psi_{2}\right) h_{2}+\nabla_{0} \mathcal{J} \nabla_{\mathbf{X}} h_{0}+\nabla_{0} \nabla_{\mathbf{X}} \nabla_{0}^{2} h_{0} \\
& +\nabla_{0}^{2}\left(\nabla_{0} \nabla_{\mathbf{X}}+\nabla_{\mathbf{X}} \nabla_{0}\right) h_{0}
\end{aligned}
$$

and explicit out the calculation on terms entering the right hand side of the above equation. Using $\partial / \partial X_{\gamma}=$ $\left(\partial q_{\alpha \beta} / \partial X_{\gamma}\right) \partial / \partial q_{\alpha \beta}=\left(\partial \psi_{\alpha} / \partial X_{\gamma} \partial X_{\beta}\right) \partial / \partial q_{\alpha \beta}$, we can write:

$$
\begin{aligned}
\nabla_{0} \mathcal{J} \nabla_{\mathbf{X}} h_{0} & =\frac{\partial \psi_{\alpha}}{\partial X_{\gamma} \partial X_{\beta}} q_{i l} \partial i\left(\mathcal{J}_{l \beta} \frac{\partial h_{0}}{\partial q_{\gamma \alpha}}\right), \\
\nabla_{0}^{2} \nabla_{0} \nabla_{\mathbf{X}} h_{0} & =\frac{\partial \psi_{\alpha}}{\partial X_{\gamma} \partial X_{\beta}} \nabla_{0}^{2} q_{i \gamma} \frac{\partial h_{i}}{\partial q_{\alpha \beta}}, \\
\nabla_{0}^{2} \nabla_{\mathbf{X}} \nabla_{0} h_{0} & =\frac{\partial \psi_{\alpha}}{\partial X_{\gamma} \partial X_{\beta}}\left(\nabla_{0}^{2} \delta_{i \alpha} \delta_{\gamma \beta} h_{i}+\nabla_{0}^{2} q_{i \gamma} \frac{\partial h_{i}}{\partial q_{\alpha \beta}}\right), \\
\nabla_{0} \nabla_{\mathbf{X}} \nabla_{0}^{2} h_{0} & =\frac{\partial \psi_{\alpha}}{\partial X_{\gamma} \partial X_{\beta}} 2 q_{i \gamma} \delta_{j \alpha} \delta_{l \beta} q_{n l} h_{i j n} \\
+ & \frac{\partial \psi_{\alpha}}{\partial X_{\gamma} \partial X_{\beta}} q_{i \gamma} \nabla_{0}^{2} \frac{\partial h_{i}}{\partial q_{\alpha \beta}},
\end{aligned}
$$

where $\delta$ 's arise from derivatives: $\delta_{s l} \delta_{p t}=\partial q_{s p} / \partial q_{l t}$.

\section{Appendix D: Diffusion coefficients for the hexagonal symmetry}

Our aim here is to prove that $D_{11}^{11} \equiv D_{22}^{22} \equiv D_{11}$ for hexagonal symmetry. We show detailed calculations in this specific case only, that can serve as a guide for other symmetries. We use definitions (26) in order to write the two coefficients $D_{\beta \gamma}^{i \alpha}$. First we write down some of the $c_{\beta \gamma}^{\alpha}$ 's that enter the diffusion coefficients:

$-c_{11}^{1}=q\left[\left(\partial_{1}+\partial_{2}\right)\left(\mathcal{J}_{11} \partial_{q} h_{0}\right)+2 \sqrt{3}\left(\partial_{1}-\partial_{2}\right)\left(\mathcal{J}_{21} \partial_{q} h_{0}\right)\right]$
$+\frac{q^{2}}{2}\left(h_{111}+2 h_{112}+h_{122}\right)+3 \nabla_{0}^{2} q\left(\partial_{1}+\partial_{2}\right) \partial_{q} h_{0}+\nabla_{0}^{2} h_{1}$,

and:

$$
\begin{aligned}
& -c_{22}^{2}=-q\left[\frac{1}{\sqrt{3}}\left(\partial_{1}+\partial_{2}\right)\left(\mathcal{J}_{12} \partial_{q} h_{0}\right)+\left(\partial_{1}-\partial_{2}\right)\left(\mathcal{J}_{22} \partial_{q} h_{0}\right)\right] \\
& +\frac{3}{2} q^{2}\left(h_{112}+h_{222}\right)-3 \nabla_{0}^{2} q\left(\partial_{1}-\partial_{2}\right) \frac{\partial h_{0}}{\partial q}+\nabla_{0}^{2} h_{2} .
\end{aligned}
$$

For the Jacobian matrix components we use condition (14), that amounts to:

$$
\mathbf{j}\left(\nabla_{0} h_{0}\right)=-\nabla_{0}\left(\nabla_{0}^{2} h_{0}\right),
$$

allowing to obtain:

$$
\begin{gathered}
\mathcal{J}_{11}=\frac{q^{2}}{2}\left[\frac{\left(h_{12}-h_{22}\right)\left(h_{1111}+h_{1222}\right)-\left(h_{11}-h_{12}\right)\left(h_{1112}+h_{2222}\right)}{h_{11} h_{22}-h_{12}^{2}}\right], \\
\mathcal{J}_{12}=\frac{q^{2}}{2 \sqrt{3}}\left[\frac{\left(h_{11}+h_{12}\right)\left(h_{1112}+h_{2222}\right)-\left(h_{12}+h_{22}\right)\left(h_{1111}+h_{1222}\right)}{h_{11} h_{22}-h_{12}^{2}}\right], \\
\mathcal{J}_{21}=\frac{\sqrt{3} q^{2}}{2}\left[\frac{\left(h_{12}-h_{11}\right)\left(h_{1112}-2 h_{1122}+2 h_{1222}-h_{2222}\right)+\left(h_{12}-h_{22}\right)\left(h_{1111}+2 h_{1122}-h_{1222}-2 h_{1112}\right)}{h_{11} h_{22}-h_{12}^{2}}\right], \\
\mathcal{J}_{22}=\frac{q^{2}}{2}\left[\frac{\left(h_{11}+h_{12}\right)\left(h_{1112}-2 h_{1122}+2 h_{1222}-h_{2222}\right)+\left(h_{22}+h_{12}\right)\left(h_{1222}+2 h_{1112}-h_{1111}-2 h_{1122}\right)}{h_{11} h_{22}-h_{12}^{2}}\right] .
\end{gathered}
$$


Moreover, $\left\langle h_{1}^{2}\right\rangle=\left\langle h_{2}^{2}\right\rangle \equiv\left\langle h_{\varphi}^{2}\right\rangle$. We can now write

$$
\begin{gathered}
D_{11}^{11}=\frac{1}{\left\langle h_{1} h_{2}\right\rangle}\left[q^{3} \partial_{q}\left\langle h_{11}^{2}\right\rangle+q^{2}\left\langle h_{11}^{2}\right\rangle+\frac{q^{2}}{2}\left\langle h_{11} h_{22}\right\rangle\right], \\
D_{22}^{22}=\frac{1}{\left\langle h_{1} h_{2}\right\rangle}\left\{4 q^{3}\left[\frac{1}{2} \partial_{q}\left\langle h_{11}^{2}\right\rangle-\frac{3}{2} \partial_{q}\left\langle h_{11} h_{12}\right\rangle+\partial_{q}\left\langle h_{11} h_{22}\right\rangle\right]+q^{2}\left[\frac{7}{2}\left\langle h_{11} h_{22}\right\rangle-\frac{1}{2}\left\langle h_{11}^{2}\right\rangle\right]\right\} .
\end{gathered}
$$

Invoking invariances under $\pi / 3$, we find that specific and non-trivial relations are verified among scalar products:

$$
\left\langle h_{11}^{2}\right\rangle=2\left\langle h_{11} h_{12}\right\rangle, \quad\left\langle h_{11} h_{12}\right\rangle=\left\langle h_{11} h_{22}\right\rangle .
$$

By means of the first relation of (D4), we finally get:

$$
D_{11}^{11}-D_{22}^{22}=\frac{1}{h_{1} h_{2}}\left(3 q^{2}+4 q^{3} \partial_{q}\right)\left[\left\langle h_{11} h_{12}\right\rangle-\left\langle h_{11} h_{22}\right\rangle\right]
$$

that vanishes thanks to the second relation (D4), therefore $D_{11}^{11}=D_{22}^{22}$.

\section{Appendix E: q-component for various symmetries}

We declare the q-vectors used in the treated cases:

- rectangular: $\quad \mathbf{q}_{1}=q(1,0), \mathbf{q}_{2}=p q(0,1)$

- square: $\mathbf{q}_{1}=q(1,0), \mathbf{q}_{2}=q(0,1)$

- hexagonal: $\quad \mathbf{q}_{1}=q / 2(1, \sqrt{3}), \mathbf{q}_{2}=q / 2(1,-\sqrt{3})$

- triangular: $\quad \mathbf{q}_{1}=q / 2(1, \sqrt{3}), \mathbf{q}_{2}=q / 2(1,-\sqrt{3})$

\section{Appendix F: Phase equation eigenvalue spectrum for the rectangular case}

The eigenvalue sign for the rectangular case spectrum in Eq. (38) is not easy to recognize because of the $\theta$ dependence. Thanks to continuity in $\theta$, however, we limit the analysis only to the extremal values, namely the $\theta$ directions such that $\partial \Omega_{1,2} / \partial \theta=0$. Let's use the notation $s \equiv \sin ^{2} \theta$ and let's define $\Omega_{1,2}(K, s)=G(s) K^{2} / 2$ with

$$
G(s)=-\left[\left(D_{11}^{11}+D_{11}^{22}\right)+A s\right] \pm \sqrt{D+B s+B s^{2}},
$$

where

$$
\begin{aligned}
& A:=D_{22}^{11}+D_{22}^{22}-D_{11}^{11}-D_{11}^{22}, \\
& B:=2\left(D_{11}^{11}-D_{11}^{22}\right)\left(D_{22}^{11}-D_{22}^{22}-D_{11}^{11}+D_{11}^{22}\right)+4 D_{12}^{12} D_{12}^{21}, \\
& C:=\left(D_{22}^{11}-D_{22}^{22}-D_{11}^{11}+D_{11}^{22}\right)^{2}-4 D_{12}^{12} D_{12}^{21}, \\
& D:=\left(D_{11}^{11}-D_{11}^{22}\right)^{2} .
\end{aligned}
$$

We now write an analogous extremal condition for Eq. (38):

$$
\frac{\partial G(s)}{\partial s} \frac{\partial s}{\partial \theta}=0
$$

The annihilation of the second factor reveals that $\theta=$ $0+n \pi$ and $\theta=\pi / 4+n \pi$ are extremal directions, the annihilation of the first factor gives further solutions:

$$
\begin{aligned}
& s=\frac{1}{2 C\left(A^{2}-C\right)}\left\{-B\left(A^{2}-C\right)\right. \\
& \left. \pm \sqrt{B^{2}\left(A^{2}-C\right)^{2}-4 C\left(A^{2}-C\right)\left(A^{2} D-B^{2} / 4\right)}\right\}
\end{aligned}
$$

A check of its sign and value, if not feasable in general terms, is possible in the limit of small amplitude for the growing pattern, as we are going to proof. Given the symmetries indicated in Table I for the rectangular patern and following the same steps indicated in Section $\mathrm{V}$ for the square symmetry case, a rectangular Fourier series is, at first order $(n, m= \pm 1)$ :

$$
\begin{aligned}
h(\mathbf{x}, t) & \simeq a_{1}(t)\left(e^{i q(x+p y)}+e^{i q(x-p y)}+c . c .\right)= \\
& =4 a(t) \cos \varphi_{1} \cos \varphi_{2}
\end{aligned}
$$

where $a_{1,1}=a_{1,-1}=a_{-1,1}=a_{-1,-1} \equiv a$. From the second expression in Eq. (F5) is straightforward to calculate the scalar products appearing in the six diffusion coefficients, obtaining the following approximated forms:

$$
\begin{aligned}
D_{11}^{11} & =\frac{1}{\left\langle h_{1}^{2}\right\rangle}\left[\partial_{q}\left(q^{3}\left\langle h_{11}^{2}\right\rangle\right)+q^{3} p^{2} \partial_{q}\left\langle h_{12}^{2}\right\rangle+q^{2} p^{2}\left\langle h_{12}^{2}\right\rangle\right] \\
& \simeq\left(3+p^{2}\right) q^{2}+\left(1+p^{2}\right) q^{3} \partial_{q}\left(a^{2}\right) / a^{2}, \\
D_{22}^{22} & =\frac{1}{\left\langle h_{2}^{2}\right\rangle}\left[p^{2} \partial_{q}\left(q^{3}\left\langle h_{22}^{2}\right\rangle\right)+q^{3} \partial_{q}\left\langle h_{12}^{2}\right\rangle+q^{2}\left\langle h_{12}^{2}\right\rangle\right] \\
& \simeq\left(3 p^{2}+1\right) q^{2}+\left(1+p^{2}\right) q^{3} \partial_{q}\left(a^{2}\right) / a^{2}, \\
D_{22}^{11} & =\frac{q^{2}}{\left\langle h_{1}^{2}\right\rangle}\left[\left\langle h_{11}^{2}\right\rangle+3 p^{2}\left\langle h_{12}^{2}\right\rangle\right] \simeq\left(3+p^{2}\right) q^{2}, \\
D_{11}^{22} & =\frac{q^{2}}{\left\langle h_{2}^{2}\right\rangle}\left[3\left\langle h_{12}^{2}\right\rangle+p^{2}\left\langle h_{22}^{2}\right\rangle\right] \simeq\left(1+3 p^{2}\right) q^{2}, \\
D_{12}^{12} & =\frac{2}{\left\langle h_{1}^{2}\right\rangle}\left[p q^{3}\left\langle h_{11} \partial_{q} h_{12}\right\rangle+p^{3} q^{3}\left\langle h_{22} \partial_{q} h_{12}\right\rangle+p q^{2}\left\langle h_{12}^{2}\right\rangle\right] \\
& \simeq 2 p q^{2}, \\
D_{21}^{12} & =\frac{2}{\left\langle h_{2}^{2}\right\rangle}\left[q^{3} / p\left\langle h_{11} \partial_{q} h_{12}\right\rangle+p q^{3}\left\langle h_{22} \partial_{q} h_{12}\right\rangle+2 p q^{2}\left\langle h_{12}^{2}\right\rangle\right] \\
& \simeq 4 p q^{2} .
\end{aligned}
$$

Let's indicate, for the sake of brevity, $m \equiv q^{3} \partial_{q}\left(a^{2}\right) / a^{2}$ and let's rewrite Eqs.(F2):

$$
\begin{aligned}
& A:=4\left(p^{2}-1\right) q^{2}, \\
& B:=-4\left(1+p^{2}\right)^{2} m^{2}+64 p^{2} q^{2}, \\
& C:=4\left(1+p^{2}\right)^{2} m^{2}-32 p^{2} q^{2}, \\
& D:=\left(1+p^{2}\right)^{2} m^{2} .
\end{aligned}
$$


In the weakly nonlinear regime Eqs. (F4) become:

$$
s=\frac{1}{2} \pm 2 \sqrt{2} \frac{q^{4}}{m^{2}} \frac{p^{2}-1}{\left(p^{2}+1\right)^{2}} p .
$$

We note that for $p=1$ (square symmetry case), these two directions coincide in $\theta=\pi / 4$. The corresponding eigenvalues can be now written in their approximated expressions; for completeness we list all the $\Omega_{1,2}(K)$ found for the rectangular case spectrum Eq. (38):

$$
\begin{aligned}
& \Omega_{1}^{0}(K, p, q) \simeq-\left(3+p^{2}\right) q^{2} K^{2}, \\
& \Omega_{2}^{0}(K, p, q) \simeq-\left(1+p^{2}\right) m K^{2}, \\
& \Omega_{1}^{\pi / 2}(K, p, q) \simeq-\left(1+3 p^{2}\right) q^{2} K^{2}, \\
& \Omega_{2}^{\pi / 2}(K, p, q) \simeq-\left(1+p^{2}\right) m K^{2}, \\
& \Omega_{1}^{s+}(K, p, q)=\Omega_{2}^{s+}(K, p, q)=\Omega_{1}^{s-}(K, p, q)=\Omega_{2}^{s-}(K, p, q)= \\
& \simeq-\left(1+p^{2}\right) m \frac{K^{2}}{2} .
\end{aligned}
$$

This weakly nonlinear analysis allows finally to recognize the positivity of $\Omega_{1}^{0}(K, p, q)$ and $\Omega_{1}^{\pi / 2}(K, p, q)$, already manifest in Eqs. (IV B), and to state how the sign of all the other eigenvalues remains undoubtedly determined by the behaviour of the stationary amplitude with respect to the stationary wavelength $\lambda=2 \pi / q$. In Section $\mathrm{V}$ a further step, explicitly shown for the square symmetry case but still valid also for the rectangular symmetry, allows also to proof that the behaviour of $\partial_{q}(a)$ is related to the specific form of the current $\mathbf{j}$.
[1] K. Huang, Statistical Mechanics, 2nd ed. (John Wiley \& Sons, 1987).

[2] A. Bray, Advances in Physics 43, 357 (1994); A. J. Bray and A. D. Rutenberg, Phys. Rev. E 49, R27 (1994).

[3] The clock models, or vector Potts models, are 2D planar spin models where the spins are restricted to $q$ evenlyspaced orientations.

[4] P. Politi and C. Misbah, Phys. Rev. E 73, 036133 (2006).

[5] S. Biagi, C. Misbah, and P. Politi, Phys. Rev. Lett. 109, 096101 (2012).

[6] P. Politi, G. Grenet, A. Marty, A. Ponchet, and J. Villain, Physics Reports 324, 271 (2000).

[7] G. Danker, O. Pierre-Louis, K. Kassner, and C. Misbah, Phys. Rev. E 68, 020601 (2003).

[8] More precisely, we should require that volume is conserved, which forbids voids and overhangs.

[9] Thermal detachment from steps and fluctuations in the diffusing current are two of them.

[10] $U(m)$ is an even potential with a minimum in $m=0$, since $j(m)=m$ at small $m$.

[11] C. Bender and S. Orszag, Advanced Mathematical Methods for Scientists and Engineers I: Asymptotic Methods and Perturbation Theory, Advanced Mathematical Methods for Scientists and Engineers (Springer, 1999).

[12] R. Hoyle, Pattern Formation: An Introduction to Methods (Cambridge University Press, 2006).

[13] D. Zwillinger, Handbook of Differential Equations, v. 1 (Academic Press, 1998).

[14] The scalar product is defined as $\langle f, g\rangle:=$ $\frac{1}{(2 \pi)^{2}} \int_{0}^{2 \pi} \int_{0}^{2 \pi} d \varphi_{1} d \varphi_{2} f^{*} g$.

[15] M. Nicoli, C. Misbah, and P. Politi, Phys. Rev. E 87, 063302 (2013).

[16] M. Kalff, P. milauer, G. Comsa, and T. Michely, Surface Science 426, L447 (1999).

[17] This is strictly true for short times, when Eq. (24) is exact.

[18] Such a case, in fact, corresponds to congruent $q$-vectors, therefore (see Tab. I) to a geometry of the pattern that resembles the square one: directions $\theta=0$ and $\theta=\pi / 2$ must be equivalent high symmetry orientations, thus corresponding eigenvalues must be equal. Restoration of a square symmetry, however, is not complete because, for the present case, we have imposed a 2-fold invariance, not a 4-fold one.

[19] C. Misbah and P. Politi, Phys. Rev. E 80, 030106 (2009).

[20] For square and rectangular symmetries, we have not been able to find explicitely this function. However, coarsening is still related to the negative sign of a suitable diffusion coefficient.

[21] For a general 6-fold Fourier series of kind $h(\mathbf{x}, t)=$ $\sum_{n, m} a_{n, m}(t) e^{i q / 2[(n+m) x+\sqrt{3}(n-m) y]}$ we find the following relations:

$$
\begin{aligned}
& a_{n, m}=a_{m, m-n}=a_{m-n,-n}= \\
& =a_{-n,-m}=a_{-m,-(m-n)}=a_{-(m-n), n}
\end{aligned}
$$

to be valid among amplitudes.

[22] In order to get the 3-fold pattern, it is enough to suitably superpose two 6-fold patterns. The value of $\mathbf{x}_{0}$ can be easily understood as the nearest neighbor distance in a honeycomb lattice, when this lattice is considered as the superposition of two 6-fold lattices.

[23] It should be stressed that in the previous formula $L$ depends on time because coarsening occurs, but the wavevector $q=2 \pi / L$ appearing in $\mathcal{D}(q)$ characterizes periodic stationary configurations.

[24] The sign of $D_{11}+D_{22}+D_{12}$ is determined by the inequality $c_{11}>c_{12}$, whose validation has to be brought back to the study of the two integrands $\left(\frac{\partial^{2} h_{0}}{\partial x^{2}}\right)^{2}$ and $\left(\frac{\partial^{2} h_{0}}{\partial x \partial y}\right)^{2}$ appearing in the scalar products. For symmetry reasons, this is equivalent to

$$
\frac{1}{2}\left[\left(\frac{\partial^{2} h_{0}}{\partial x^{2}}\right)^{2}+\left(\frac{\partial^{2} h_{0}}{\partial y^{2}}\right)^{2}\right] \geq\left(\frac{\partial^{2} h_{0}}{\partial x \partial y}\right)^{2}
$$


For a general 4-fold Fourier series as $h(\mathbf{x}, t)=$ $\sum_{n, m} a_{n, m}(t) e^{i q(n x+m y)}$, this is nothing but

$$
\frac{1}{2}\left(n^{4}+m^{4}\right) \geq n^{2} m^{2}
$$

alias $\left(n^{2}-m^{2}\right)^{2} \geq 0$, thus verifying the inequality.

[25] The case $c_{11}=c_{12}$ would produce a one-dimensional dynamics, thus we will not take it into consideration.

[26] P. Politi and A. Torcini, The European Physical Journal B - Condensed Matter and Complex Systems 53, 401 (2006).

[27] J. Langer, Annals of Physics 65, 53 (1971).

[28] T. Kawakatsu and T. Munakata, Progress of Theoretical Physics 74, 11 (1985); K. Kawasaki and T. Ohta, Physica A: Statistical Mechanics and its Applications 116, 573 (1982).

[29] We do not make a stronger claim because we limit the comparison to $d=1$ and $d=2$.

[30] C. Misbah, O. Pierre-Louis, and Y. Saito, Rev. Mod. Phys. 82, 981 (2010).
[31] L. Golubović, A. Levandovsky, and D. Moldovan, East Asian Journal on Applied Mathematics 1, 297 (2001).

[32] T. Michely and J. Krug, Islands, Mounds, and Atoms: Patterns and Processes in Crystal Growth Far from Equilibrium, Physics and astronomy online library (Springer Berlin Heidelberg, 2004).

[33] R. V. Kohn and X. Yan, Communications on Pure and Applied Mathematics 56, 1549 (2003).

[34] B. Li, Nonlinearity 19, 2581 (2006).

[35] S. J. Watson and S. A. Norris, Phys. Rev. Lett. 96, 176103 (2006).

[36] L. Golubović, Phys. Rev. Lett. 78, 90 (1997).

[37] D. Moldovan and L. Golubovic, Phys. Rev. E 61, 6190 (2000).

[38] A. Levandovsky and L. Golubović, Phys. Rev. B 69, 241402 (2004).

[39] M. Siegert, Phys. Rev. Lett. 81, 5481 (1998).

[40] J. A. Stroscio, D. T. Pierce, M. D. Stiles, A. Zangwill, and L. M. Sander, Phys. Rev. Lett. 75, 4246 (1995).

[41] P. Politi, Phys. Rev. E 58, 281 (1998).

[42] P. Politi and J. Krug, Surface Science 446, 89 (2000).

[43] A. J. Bray, Phys. Rev. Lett. 62, 2841 (1989).

[44] M. Siegert, Physica A: Statistical Mechanics and its Applications 239, 420 (1997). 